\title{
Numerical Quadrature and Nonlinear Sequence Transformations; Unified Rules for Efficient Computation of Integrals With Algebraic and Logarithmic Endpoint Singularities
}

\author{
By Avram Sidi
}

\begin{abstract}
Some nonlinear transformations for accelerating the convergence of infinite sequences due to Levin are reviewed, and new results of practical importance in applications are given. Using these results, the transformations of Levin are modified and used to obtain new numerical integration formulas for weight functions with algebraic and logarithmic endpoint singularities, which are simpler to compute and practically as efficient as the corresponding Gaussian formulas. They also have the additional advantage that different weight functions of a certain type can have the same set of abscissas associated with them. It is shown that the formulas obtained are of interpolatory type. Furthermore, for some cases it is proved that the abscissas are in the interval of integration, although numerical results indicate that this is so in all cases and that the weights are all positive. Several numerical examples that illustrate the high accuracy and convenience of the new formulas are appended.
\end{abstract}

1. Introduction and Motivation. Let $f(z)$ be a function which is analytic in a simply connected open set $G$ of the complex.z-plane, which contains the finite real interval $[a, b]$. Also, let $\Gamma$ be a closed Jordan curve in $G$, containing $[a, b]$ in its interior. Then from Cauchy's theorem

$$
f\left(z_{0}\right)=\frac{1}{2 \pi_{i}} \int_{\Gamma} \frac{f(z)}{z-z_{0}} d z
$$

whenever $z_{0}$ is in the interior of $\Gamma$. Using (1.1), it is possible to express the integral

$$
I[f]=\int_{a}^{b} w(x) f(x) d x,
$$

whenever $w(x)$ is an integrable function on $[a, b]$, as

$$
I[f]=\frac{1}{2 \pi i} \int_{\Gamma} H(z) f(z) d z,
$$

where

$$
H(z)=\int_{a}^{b} \frac{w(x)}{z-x} d x
$$

The function $H(z)$ is analytic in the $z$-plane cut along $[a, b]$ and for sufficiently large

Received December 21, 1978; revised June 25, 1979.

1980 Mathematics Subject Classification. Primary 41A55, 41A20, 40A05, 40A25, 41 A60. 
$z$ has the expansion

$$
H(z)=\sum_{n=1}^{\infty} \frac{\mu_{n}}{z^{n}}
$$

where

$$
\mu_{n}=\int_{a}^{b} w(x) x^{n-1} d x, \quad n=1,2, \ldots
$$

Let $x_{k, n}, n=1,2, \ldots, k$, be $k$ distinct points and let

$$
I_{k}[f]=\sum_{n=1}^{k} A_{k, n} f\left(x_{k, n}\right)
$$

be a numerical quadrature formula. Assuming that $x_{k, n}$ are all in the interior of $\Gamma$ and using (1.1), we can express (1.7) in the form

$$
I_{k}[f]=\frac{1}{2 \pi i} \int_{\Gamma} H_{k}(z) f(z) d z,
$$

where

$$
H_{k}(z)=\sum_{n=1}^{k} \frac{A_{k, n}}{z-x_{k, n}} .
$$

Now the error in the numerical quadrature formula (1.7) can be expressed as

$$
E_{k}[f]=I[f]-I_{k}[f]=\frac{1}{2 \pi i} \int_{\Gamma}\left[H(z)-H_{k}(z)\right] f(z) d z .
$$

From (1.10) we trivially obtain

$$
\left|E_{k}[f]\right| \leqslant \frac{1}{2 \pi} \max _{\zeta \in \Gamma}|f(\zeta)| \int_{\Gamma}\left|H(z)-H_{k}(z)\right||d z| .
$$

It is clear from (1.11) that if $\left|H(z)-H_{k}(z)\right|$ is small on $\Gamma$, then $\left|E_{k}[f]\right|$ will be small too. Furthermore, if $H(z)-H_{k}(z) \rightarrow 0$ uniformly on $\Gamma$ as $k \rightarrow \infty$, then $E_{k}[f] \rightarrow$ 0 too. Since we want the error to go to zero for all $f(z)$ analytic on $[a, b]$ and, hence, in some region containing $[a, b]$, we should actually demand that $H(z)-$ $H_{k}(z) \rightarrow 0$ uniformly in the complement of any open set $G$ that contains in its interior the branch cut of $H(z)$, i.e., the line segment $[a, b]$; this is what we shall mean below by uniform convergence of $H_{k}(z)$ to $H(z)$. If this convergence is also quick, then $E_{k}[f] \rightarrow 0$ quickly too. The uniform convergence of $H_{k}(z)$ (in the above sense) may be very critical as we now demonstrate by considering two well-known numerical quadrature methods.

(1) Numerical quadrature formulas of interpolatory type: For these formulas

$$
H(z)-H_{k}(z)=O\left(\frac{1}{z^{k+1}}\right) \quad \text { as } z \rightarrow \infty,
$$

where the abscissas $x_{k, n}$ are preassigned. (1.12) can be proved easily by recalling that

$$
I_{k}\left[x^{n}\right]=I\left[x^{n}\right], \quad n=0,1, \ldots, k-1,
$$


and expanding $H_{k}(z)$ in inverse powers of $z$ and comparing with (1.5) with the help of (1.13). It turns out that $H_{k}(z)$ is not a very good approximation to $H(z)$ for small $z$ when $x_{k, n}$ are arbitrarily assigned. In fact, for Newton-Cotes formulas in which $x_{k, n}=$ $a+(n-1)(b-a) /(k-1), n=1, \ldots, k, I_{k}[f]$ may diverge as $k \rightarrow \infty$, even when $f(z)$ is analytic on a simply connected open set $G$ containing $[a, b]$ in its interior, see Pólya (1933) and Davis (1955). This fact together with (1.10) shows that, for this choice of the $x_{k, n}, H_{k}(z)$ does not converge to $H(z)$ uniformly in $z$ (in the above sense). We thus conclude that $E_{k}(f)$ may diverge if $H_{k}(z)$ does not converge to $H(z)$ uniformly, even for analytic $f(z)$.

(2) Numerical quadrature formulas of Gaussian type: For these formulas

$$
H(z)-H_{k}(z)=O\left(\frac{1}{z^{2 k+1}}\right) \quad \text { as } z \rightarrow \infty,
$$

where the abscissas $x_{k, n}$ are not preassigned. $H_{k}(z)$ in this case is simply the $((k-1) / k)$ Padé approximant to the series (1.5). The proof of (1.14) is similar to that of (1.12). If $w(x) \geqslant 0$ on $[a, b]$ and $K(x)=\int_{a}^{x} w(t) d t$ is strictly increasing on $[a, b]$, then all the $x_{k, n}$ are distinct and are in $(a, b)$. Besides all the $A_{k, n}$ are positive, see Davis and Rabinowitz (1975, p. 74). It is also known that, as $k \rightarrow \infty, H_{k}(z)$ converges to $H(z)$ uniformly in $z$ (in the above sense), see Baker (1975, Chapter 16). Indeed, the convergence of $H_{k}(z)$ to $H(z)$ is very quick, which accounts for the high accuracy of $I_{k}[f]$ even for moderate $k$.

Our purpose in this work is to use a modification of some nonlinear sequence transformations due to Levin (1973) to obtain new rational approximations $H_{k}(z)$ to $H(z)$ and use these to derive new numerical quadrature formulas. There is ample numerical evidence that suggests that the $H_{k}(z)$ obtained this way converge to $H(z)$ uniformly (in the above sense) and also very quickly as $k \rightarrow \infty$, see e.g. Longman (1973), and this suggests that they could be used to develop numerical quadrature formulas of high accuracy, provided of course, that the $x_{k, n}$ are all real and distinct and lie on $[a, b]$ and that the $A_{k, n}$ are all positive. It seems that both of these conditions are satisfied for some useful weights $w(x)$. Although these numerical quadrature formulas are not derived by imposing conditions like (1.13), they do give rise to relations similar to them. They also have the very interesting property that their abscissas are the same for certain classes of weights $w(x)$, e.g., for the integral $\int_{0}^{1} x^{\beta} f(x) d x, \beta>-1$, the abscissas are the same for all $\beta$. Another property which makes these formulas useful is that the approximations $I_{k}[f]$ are practically as good as those obtained using the corresponding Gaussian rules. In addition, the computation of the abscissas is simpler for the new rules than for the corresponding Gaussian rules, since the polynomials that give them are readily available.

2. Levin's Transformations and Some Recent Results. In this section we briefly review Levin's transformations and some recent developments due to the present author, see Sidi (1979), which are crucial for understanding when these transformations work and why they work so well. 
Let $A_{0}=0, A_{r}, r=1,2, \ldots$, be an infinite sequence (convergent or not) whose limit or antilimit in the language of Shanks (1955) and Levin (1973) is $A$. Then the approximation $T_{k, n}$ to $A$ together with the parameters $\gamma_{i}, i=0,1, \ldots, k-1$, is defined by the equations

$$
A_{r-1}=T_{k, n}+R_{r} \sum_{i=0}^{k-1} \gamma_{i} / r^{i}, \quad r=n, n+1, \ldots, n+k,
$$

where $R_{r}$ will be specified below. The solution to these equations is simply

$$
T_{k, n}=\frac{\sum_{j=0}^{k}(-1)^{j}\left({ }_{j}^{k}\right)(n+j)^{k-1} A_{n+j-1} / R_{n+j}}{\sum_{j=0}^{k}(-1)^{j}\left({ }_{j}^{k}\right)(n+j)^{k-1} / R_{n+j}} .
$$

The equations in (2.1) and, hence, the expression for $T_{k, n}$ in (2.2) are slightly different from those given by Levin in that Levin writes $A_{r}$ on the left-hand side of (2.1) instead of $A_{r-1}$ and, hence, $A_{n+j}$ instead of $A_{n+j-1}$ in the numerator of (2.2). Now for the $t$-transformation of Levin $R_{1}=A_{1}, R_{r}=A_{r}-A_{r-1}, r \geqslant 2$, and for the $u$-transformation of Levin $R_{1}=A_{1}, R_{r}=r\left(A_{r}-A_{r-1}\right), r \geqslant 2$. For these two transformations it is easy to show that the definition in (2.1) and that of Levin give identical results for $T_{k, n}$. However, for our purposes in this work it is more appropriate to use (2.1). We shall say more on this later.

The convergence properties of $T_{k, n}$ have been partially studied by the present author, Sidi (1979), and some convergence theorems for two limiting processes, namely, (1) $k$ fixed, $n \rightarrow \infty$, (2) $n$ fixed, $k \rightarrow \infty$, have been proved. The analysis in the above work and also numerical experience suggest the following:

(1) $T_{k, n}$ is a good approximation to $A$ (limit or antilimit) and converges to $A$ very quickly as $k \rightarrow \infty$ when $A_{r}$ is of the form

$$
A_{r}=A+R_{r} f(r), \quad r=1,2,3, \ldots,
$$

where $f(x)$, when considered as a function of the continuous variable $x$, has an asymptotic expansion of the form

$$
f(x) \sim \beta_{0}+\frac{\beta_{1}}{x}+\frac{\beta_{2}}{x^{2}}+\cdots, \quad \text { as } x \rightarrow \infty,
$$

and is an infinitely differentiable function of $x$ up to $x=\infty$. When $A_{r}$ is not of this form, then $T_{k, n}$ is useless. Therefore, it seems that conditions (2.3) and (2.4) are the most crucial for the quick convergence of $T_{k, n}$ as $k \rightarrow \infty$.

(2) If we define $\bar{R}_{r}=R_{r} g(r)$, where $g(x)$ has the same properties as $f(x)$ and $\lim _{x \rightarrow \infty} g(x) \neq 0$, then we can write (2.3) in the form

$$
A_{r}=A+\bar{R}_{r} \bar{f}(r), \quad r=1,2,3, \ldots,
$$

where $\bar{f}(x)=f(x) / g(x)$ and $\bar{f}(x)$ has the same properties as $f(x)$. Therefore, judging from (1), the exact form of $R_{r}$ in (2.2) is not important, and $R_{r}$ can be replaced by $\bar{R}_{r}$.

(3) For $k \rightarrow \infty$, it does not make much difference if $R_{r}$ in (2.2) is replaced by $r R_{r}$ where $s$ is a positive integer which is not too large. However, $s<0$ may destroy the accuracy of $T_{k, n}$. This is in agreement with the numerical results of Levin (1973) which 
show that the $u$-transformation is efficient when the $t$-transformation is, but the opposite is not true.

The following theorem, whose detailed proof can be found in Sidi (1979), gives sufficient conditions for (2.3) and (2.4) to hold for the case of a convergent sequence. This theorem is a special case of a more general theorem due to Levin and Sidi (1975).

(4) THEOREM 2.1. Let the sequence $A_{r}=\sum_{j=1}^{r} a_{j}, r=1,2, \ldots$, be such that the terms $a_{r}$ satisfy a linear first-order homogeneous difference equation of the form

$$
a_{r}=p(r) \Delta a_{r}, \quad r=1,2, \ldots,
$$

where $\Delta a_{r}=a_{r+1}-a_{r}$ and $p(x)$, considered as a function of the continuous variable $x$ has, as $x \rightarrow \infty$, an asymptotic expansion of the form

$$
p(x) \sim x^{i}\left(p_{0}+\frac{p_{1}}{x}+\frac{p_{2}}{x^{2}}+\cdots\right),
$$

for $i$ an integer $\leqslant 1$. Let $\lim _{r \rightarrow \infty} A_{r}=A$ exist. Assume that $\lim _{r \rightarrow \infty} p(r) a_{r}=0$ and that $l \bar{p} \neq 1, l=-1,1,2,3, \ldots$, where $\bar{p}=\lim _{x \rightarrow \infty} p(x) / x$. Then $A-A_{r-1}=\Sigma_{j=r}^{\infty} a_{j}$ has, as $r \rightarrow \infty$, an asymptotic expansion of the form

$$
A-A_{r-1}=\sum_{j=r}^{\infty} a_{j} \sim \dot{r}^{i} a_{r}\left(\beta_{0}+\frac{\beta_{1}}{r}+\frac{\beta_{2}}{r^{2}}+\cdots\right) .
$$

Remark. The condition $\bar{l} \neq-1,1,2, \ldots$, seems to be satisfied for all convergent sequences.

We see from (2.7) that a very natural choice for $R_{r}$ is $r^{i} a_{r}$ and this, with $i=0$ and $i=1$, gives Levin's $t$ - and $u$-transformations, respectively.

We now give another result which is a special case of a general result due to Levin and Sidi (1975).

(5) If the numbers $a_{r} r=1,2, \ldots$, satisfy (2.5) and (2.6), then the numbers $a_{r} x^{r-1}, r=1,2, \ldots$, satisfy similar relations. Therefore, if we define $A_{r}=\Sigma_{j=1}^{r} a_{j} x^{j-1}$, $r=1,2, \ldots$, and $\lim _{r \rightarrow \infty} A_{r}=A$ exists then Theorem 2.1 applies and $A-A_{r-1}$ has an asymptotic expansion like that in (2.7) so that, by (1) we expect $T_{k, n}$ to converge to $A$ very quickly as $k \rightarrow \infty$. (Experience shows that the same $T_{k, n}$ converges quickly to the analytic continuation of $\sum_{j=1}^{\infty} a_{j} x^{j-1}$ when $x$ is outside the circle of convergence.)

We state here that all of what has been said in (1)-(5) is important in the development of the numerical quadrature formulas which we take up in Section 4.

Before we close this section we shall give two results that will be of use later in this work.

THEOREM 2.2. If $A_{0}=0, A_{r}=\Sigma_{j=1}^{r} a_{j} / z^{j}, r=1,2, \ldots$, and $R_{r}=c_{r} / z^{r}$, where $c_{r}$ is independent of $z$, then

$$
T_{k, n}=A_{n+k-1}+O\left(\frac{1}{z^{n+k}}\right) \quad \text { as } z \rightarrow \infty
$$

and the coefficient $C$ of $1 / z^{n+k}$ in the Maclaurin series of $T_{k, n}$ is given by 


$$
C=-\frac{\sum_{j=0}^{k-1}(-1)^{j}\left(\begin{array}{l}
j \\
j
\end{array}\right)(n+j)^{k-1} a_{n+j} / c_{n+j}}{(-1)^{k}(n+k)^{k-1} / c_{n+k}} .
$$

Proof. Let us subtract $A_{n+k-1}$ from $T_{k, n}$. We obtain

(L.10) $T_{k, n}-A_{n+k-1}=\frac{\sum_{j=0}^{k-1}(-1)^{j}\left({ }_{j}^{k}\right)(n+j)^{k-1}\left(A_{n+j-1}-A_{n+k-1}\right) / R_{n+j}}{\sum_{j=0}^{k}(-1)^{j}\left({ }_{j}^{k}\right)(n+j)^{k-1} / R_{n+j}}$.

Now

$$
\left(A_{n+j-1}-A_{n+k-1}\right) / R_{n+j}=-\left[\left(a_{n+j} / c_{n+j}\right)+O\left(z^{-1}\right)\right] \quad \text { as } z \rightarrow \infty,
$$

for $0 \leqslant j \leqslant k-1$. Therefore, the numerator of $(2.10)$ is simply

$$
N_{k, n}=-\left[\sum_{j=0}^{k-1}(-1)^{j}\left(\begin{array}{l}
k \\
j
\end{array}\right)(n+j)^{k-1}\left(a_{n+j} / c_{n+j}\right)+O\left(z^{-1}\right)\right] \quad \text { as } z \rightarrow \infty .
$$

Similarly, the denominator of $T_{k, n}$ is

$$
D_{k, n}=z^{n+k}\left[(-1)^{k}(n+k)^{k-1} / c_{n+k}+O\left(z^{-1}\right)\right] \quad \text { as } z \rightarrow \infty .
$$

Combining (2.11) with (2.12) in (2.10), we obtain (2.8) and (2.9).

COROLLARY. If $c_{r}=q r^{s} a_{r}$, where $q$ is a constant, $s$ is an integer, and $0 \leqslant s \leqslant$ $k-1$, then $C=a_{n+k}$ and hence

$$
T_{k, n}=A_{n+k}+O\left(\frac{1}{z^{n+k+1}}\right) \quad \text { as } z \rightarrow \infty .
$$

Proof. If we let $c_{r}=q r^{s} a_{r}$ in (2.9), we obtain

$$
C=-a_{n+k} \frac{\sum_{j=0}^{k-1}(-1)^{j}\left({ }_{j}^{k}\right)(n+j)^{k-1-s}}{(-1)^{k}(n+k)^{k-1-s}} .
$$

Now

$$
\sum_{j=0}^{k}(-1)^{j}\left(\begin{array}{l}
k \\
j
\end{array}\right)(n+j)^{l}=(-1)^{k} \Delta^{k}\left(n^{l}\right)=0, \quad l=0,1, \ldots, k-1 .
$$

Therefore,

$$
\sum_{j=0}^{k-1}(-1)^{j}\left(\begin{array}{l}
k \\
j
\end{array}\right)(n+j)^{l}=-(-1)^{k}\left(\begin{array}{l}
k \\
k
\end{array}\right)(n+k)^{l}, \quad l=0,1, \ldots, k-1 .
$$

Using this last equality in (2.14) the result follows.

Remark. The corollary above applies to Levin's $t$ - and $u$-transformations.

LEMMA 2.1. Let $H(z)$ and $\mu_{j}$ be as in (1.4)-(1.6), and let

$$
A_{0}=0, \quad A_{r}=\sum_{j=1}^{r} \mu_{j} / z^{j}, \quad r=1,2, \ldots
$$

Then

$$
H(z)=A_{r-1}+\frac{1}{z^{r}} \int_{a}^{b} \frac{w(x) x^{r-1}}{1-x / z} d x .
$$


Proof. (2.16) follows by using the identity

$$
\frac{1}{z-x}=\sum_{j=1}^{r-1} \frac{x^{j-1}}{z^{j}}+\frac{x^{r-1} / z^{r}}{1-x / z}
$$

in (1.4) and then noting (1.6).

THEOREM 2.3. Let the sequence $A_{r}, r=0,1, \ldots$, be as in Lemma 2.1 and define the polynomials $D(z)$ and $N(z)$ by

$$
D(z)=\sum_{j=0}^{k} \lambda_{j} z^{n+j-1}
$$

and

$$
N(z)=\sum_{j=0}^{k} \lambda_{j} z^{n+j-1} A_{n+j-1},
$$

where $\lambda_{j}$ are constants. Then

$$
N(z)=\int_{a}^{b} w(x) \frac{D(z)-D(x)}{z-x} d x
$$

and

$$
H(z)-\frac{N(z)}{D(z)}=\frac{Q(z)}{D(z)}
$$

where

$$
Q(z)=\int_{a}^{b} w(x) \frac{D(x)}{z-x} d x
$$

Proof. The proof of (2.20) follows by multiplying (2.16), with $r=n+j$, by $\lambda_{j} z^{n+j-1}$, and then summing over $j$ from 0 to $k$. (2.19) now follows easily from (2.20), (2.21), and (1.4).

With the help of Theorem 2.3 above we can now give an exact expression for the error in $T_{k, n}$ as applied to the partial sums of $H(z)$.

THEOREM 2.4. Let the sequence $A_{r}, r=0,1,2, \ldots$, be as in Lemma 2.1; and let $R_{r}=c_{r} / z^{r-1}$ in the expression (2.2) for $T_{k, n}$. Then $T_{k, n}=N_{k, n}(z) / D_{k, n}(z)$, where $D_{k, n}(z)$ and $N_{k, n}(z)$ are exactly of the form (2.17) and (2.18) with

$$
\lambda_{j}=(-1)^{j}\left(\begin{array}{l}
k \\
j
\end{array}\right)(n+j)^{k-1} / c_{n+j}, \quad j=0,1, \ldots, k .
$$

Therefore,

$$
N_{k, n}(z)=\int_{a}^{b} w(x) \frac{D_{k, n}(z)-D_{k, n}(x)}{z-x} d x
$$

and

$$
H(z)-T_{k, n}=\frac{Q_{k, n}(z)}{D_{k, n}(z)},
$$


where

$$
Q_{k, n}(z)=\int_{a}^{b} w(x) \frac{D_{k, n}(x)}{z-x} d x
$$

3. Asymptotic Expansions for $H(z)$. Let us consider the expansion (1.5) for $H(z)$, and define

$$
A_{0}=0, \quad A_{r}=\sum_{m=1}^{r} \frac{\mu_{m}}{z^{m}}, \quad r=1,2, \ldots
$$

In view of what has been said in the previous section we expect $T_{k, n}$ to converge to $H(z)$ as $k \rightarrow \infty$ if the $\mu_{m}$ satisfy (2.5) and (2.6). It turns out the moments of many important weight functions do satisfy (2.5) and (2.6). These weight functions and their moments are given in Table 3.1 below. (The finite interval $[a, b]$ is taken to be $[0,1]$.) In the rest of this work we shall deal only with these weights. This is not a limitation as far as practice is concerned, since these weights contain the most important algebraic and logarithmic endpoint singularities that one comes across in much of scientific work.

TABLE 3.1

\begin{tabular}{rl}
\hline$w(x)$ & \multicolumn{1}{c}{$\mu_{m}\left(=\int_{0}^{1} w(x) x^{m-1} d x\right)$} \\
\hline 1 & $1 / m$ \\
$x^{\beta}$ & $1 /(m+\beta), \beta>-1$ \\
$(1-x)^{\alpha} x^{\beta}$ & $\alpha !(m+\beta-1) ! /(m+\alpha+\beta) !, \alpha>-1, \beta>-1$ \\
$(-\log x)^{\nu}$ & $\nu ! / m^{\nu+1}, \nu>-1$ \\
$x^{\beta}(-\log x)^{\nu}$ & $\nu ! /(m+\beta)^{\nu+1}, \beta>-1, \nu>-1$ \\
$(1-x)^{\alpha} x^{\beta}(-\log x)^{\nu}$ & not known explicitly for general $\alpha, \beta, \nu$, \\
& $\alpha+\nu>-1, \beta>-1$
\end{tabular}

For the first five moments in Table 3.1 it is easy to show that (2.5) and (2.6) are satisfied. For this we write the identity

$$
\mu_{m}=h(m) \Delta \mu_{m}, \quad m=1,2, \ldots,
$$

where $h(m)=\left[\mu_{m+1} / \mu_{m}-1\right]^{-1}$ has an asymptotic expansion of the form

$$
h(m) \sim m \sum_{i=0}^{\infty} \frac{h_{i}}{m^{i}} \text { as } m \rightarrow \infty .
$$

For the sixth moment, for which an explicit simple expression is not known to the author, we can still show that (2.5) and (2.6) are satisfied and this will be done later.

Now for all the weight functions in Table 3.1 the sequence in (3.1) converges to $H(z)$ for $|z|>1$. Therefore, we can make use of Theorem 2.1 of the previous section to conclude that an asymptotic expansion like that in (2.7) exists for $|z|>1$. However, for $|z|<1$, since $\lim _{r \rightarrow \infty} A_{r}$ does not exist, we cannot say off-hand that an asymptotic expansion like that in (2.7) exists. Nevertheless, such an asymptotic expansion does exist as we show below: 
THEOREM 3.1. Consider the weight function $w(x)=(1-x)^{\alpha} x^{\beta}(-\log x)^{\nu}, \alpha+\nu>$ $-1, \beta>-1$, on $[a, b]=[0,1]$. Then for every $z$ not on the cut $[0,1], H(z)-A_{r-1}$ is of the form

$$
A_{r-1}=H(z)+R_{r} f(r)
$$

where

$$
R_{r}=1 /\left(r^{\alpha+\nu+1} z^{r}\right)
$$

and $f(r)$ is as described in (1) of Section 2.

Proof. Using the result of Lemma 2.1, we have

$$
H(z)-A_{r-1}=\frac{1}{z^{r}} \int_{0}^{1}(1-x)^{\alpha} x^{\beta}(-\log x)^{\nu} x^{r-1} \frac{d x}{1-x / z} .
$$

Making the change of variable $x=e^{-t}$ in the integral above, we obtain

$$
H(z)-A_{r-1}=\frac{1}{z^{r}} \int_{0}^{\infty} e^{-(r+\beta) t} t^{\nu}\left(1-e^{-t}\right)^{\alpha} \frac{d t}{1-e^{-t / z}} .
$$

Now $\left(1-e^{-t}\right)^{\alpha}=t^{\alpha}\left[\left(1-e^{-t}\right) / t\right]^{\alpha}$, and it can be shown easily that the function $\left[\left(1-e^{-t}\right) / t\right]^{\alpha}$ has a convergent Maclaurin series expansion. Actually, $\left[\left(1-e^{-t}\right) / t\right]^{\alpha}=$ $1+O(t)$ as $t \rightarrow 0+$. Letting $g(t)=\left[\left(1-e^{-t}\right) / t\right]^{\alpha} e^{-\beta t} /\left(1-e^{-t} / z\right)$, we express (3.7) in the form

$$
H(z)-A_{r-1}=\frac{1}{z^{r}} \int_{0}^{\infty} e^{-r t} t^{\alpha+\nu} g(t) d t
$$

Now $g(t)$ has a convergent Maclaurin series too which we denote by $g(t)=\sum_{i=0}^{\infty} g_{i} t^{i}$, with $g(0)=z /(z-1) \neq 0$. Using Watson's lemma in (3.8), see Olver $(1974$, p. 71$)$, we then have

$$
H(z)-A_{r-1} \sim \frac{1}{r^{\alpha+\nu+1} z^{r}} \sum_{i=0}^{\infty} g_{i} \frac{(\alpha+\nu+i) !}{r^{i}} \text { as } r \rightarrow \infty,
$$

which is of the form (3.4) with $R_{r}$ as in (3.5) and

$$
f(r) \sim-\sum_{i=0}^{\infty} g_{i} \frac{(\alpha+\nu+i) !}{r^{i}} \text { as } r \rightarrow \infty
$$

This completes the proof of the theorem.

We can in a similar manner show that

$$
\mu_{r}=\int_{0}^{1}(1-x)^{\alpha} x^{\beta}(-\log x)^{\nu} x^{r-1} d x \sim \frac{1}{r^{\alpha+\nu+1}} \sum_{i=0}^{\infty} \frac{l_{i}}{r^{i}} \text { as } r \rightarrow \infty .
$$

Using (3.10), it is now easy to show that (3.2) and (3.3) are also satisfied.

4. Numerical Quadrature Formulas. In this section we shall be concerned with numerical quadrature formulas for the integral

$$
I[f]=\int_{0}^{1}(1-x)^{\alpha} x^{\beta}(-\log x)^{\nu} f(x) d x, \quad \alpha+\nu>-1, \beta>-1 .
$$


Letting $R_{r}=1 /\left(r^{\alpha+\nu+1} z^{r}\right), A_{r}=\Sigma_{j=1}^{r} \mu_{j} / z^{j}, r=1,2, \ldots$, and $A_{0}=0$ in (2.2), we obtain

$$
T_{k, n}=\frac{\sum_{j=0}^{k}(-1)^{j}\left(\begin{array}{c}
k \\
j
\end{array}\right)(n+j)^{k-1}\left[(n+j)^{\alpha+\nu+1} z^{n+j}\right] \sum_{m=1}^{n+j-1} \mu_{m} / z^{m}}{\sum_{j=0}^{k}(-1)^{j}\left(\begin{array}{l}
k \\
j
\end{array}\right)(n+j)^{k-1}\left[(n+j)^{\alpha+\nu+1} z^{n+j}\right]}
$$

As we can see from (4.2), $T_{k, n}$ is a rational approximation whose numerator is $N_{k, n}(z)=$ (polynomial of degree $\leqslant n+k-2$ ) and whose denominator is $D_{k, n}(z)=$ $z^{n-1} \times$ (polynomial of degree $k$ ), so that (degree of denominator) $\geqslant$ (degree of numerator) +1 , a property that $H_{k}(z)$ is required to have; see (1.9). $H_{k}(z)$ is also required to have simple poles; this implies that we must take $n=1$ or $n=2$. Otherwise, there is a multiple pole at $z=0$. In this and the next sections we shall discuss the case $n=$ 1 only; the case $n=2$ will be discussed in Section 6 . For the case $n=1$ we, therefore, have

$$
H_{k}(z)=T_{k, 1}=\frac{\sum_{j=1}^{k}(-1)^{j}\left({ }_{j}^{k}\right)(j+1)^{k+\alpha+\nu} z^{j} \sum_{m=1}^{j} \mu_{m} / z^{m}}{\sum_{j=0}^{k}(-1)^{j}\left({ }_{j}^{k}\right)(j+1)^{k+\alpha+\nu_{z}^{j}}} .
$$

Of course we should make sure that the poles of $T_{k, 1}$ are all simple and lie on $[0,1]$. We shall show below that for $\alpha+\nu$ a nonnegative integer this property holds.

Assuming that the poles $x_{k, j}$ of $T_{k, 1}$ are all simple and lie on $[0,1]$, we can expand $T_{k, 1}$ in partial fractions as follows:

$$
H_{k}(z)=T_{k, 1}=\sum_{j=1}^{k} \frac{A_{k, j}}{z-x_{k, j}},
$$

where $A_{k, j}$ are given simply by

$$
A_{k, j}=\frac{N_{k, 1}\left(x_{k, j}\right)}{D_{k, 1}^{\prime}\left(x_{k, j}\right)}, \quad j=1,2, \ldots, k,
$$

where ' denotes differentiation with respect to $z$. We then define our numerical quadrature formula by $I_{k}[f]=\sum_{j=1}^{k} A_{k, j} f\left(x_{k, j}\right)$.

This formula has several advantages over the Gaussian integration formulas, which we now explain:

(1) The abscissas of the Gaussian integration rules are the zeros of the polynomials orthogonal with respect to $w(x)$ on the interval of integration, and their weights can also be expressed in terms of these polynomials; and all methods of computing Gaussian integration rules make direct or indirect use of the orthogonal polynomials and, thus, require their generation if they are not known. These polynomials can be generated by using their 3-term recursion relation. Given the 3-term recursion relation one can use, for example, the algorithm of Golub and Welsch (1969), to compute the abscissas and the weights. When this recursion relation is not known explicitly it can be computed, for example, by using the methods of Gautschi (1968), (1970). In one of these methods one computes by some approximate quadrature rule the coefficients of the 3-term recursion relation of the orthogonal polynomials. If this method 
is applied to a weight function of the form $w(x)=x^{\beta}(-\log x)^{\nu}$ for general $\nu$, the convergence may be rather slow. In the second method one makes use of some modified moments of $w(x)$ whose computation can again become difficult when $w(x)=$ $x^{\beta}(-\log x)^{\nu}$ for general $\nu$, for example. In our new integration rules the polynomials that give the abscissas are readily available, and the weights can be computed from Eq. (4.5).

(2) The denominator of $T_{k, 1}$ (and of $T_{k, n}$, any $n$ ) is independent of $\beta$ and depends on $\alpha$ and $\nu$ through $\alpha+\nu$. Therefore, the poles $x_{k, j}$ of $T_{k, 1}$ are independent of $\beta$ and depend only on $\alpha+\nu$. This implies that for all $\beta>-1$ and all $\alpha, \nu$ such that $\alpha+\nu>-1$ is fixed, our numerical quadrature formulas have the same abscissas, and only their weights are different. But the determination of the weights can be accomplished trivially with the help of (4.5). No such property exists for the Gaussian integration formulas. (This property does not exist if, instead of taking $R_{r}=$ $1 /\left(r^{\alpha+\nu+1} z^{r}\right)$, we take $R_{r}=\mu_{r} / z^{r}$, like in the $t$-transformation of Levin.)

(3) In view of (3) of Section 2 we can replace $R_{r}=1 /\left(r^{\alpha+\nu+1} z^{\eta}\right)$ by $R_{r}=$ $r /\left(r^{\alpha+\nu+1} z^{r}\right)$, where $s$ is a nonnegative integer which is not too large $(s=1,2$, for example), and $T_{k, n}$ will still be a very good approximation to $H(z)$. This implies that the abscissas for the weight $w(x)=(1-x)^{\alpha^{\prime}} x^{\beta^{\prime}}(-\log x)^{\nu^{\prime}}$, where $\alpha^{\prime}+\nu^{\prime}=\alpha+$ $\nu-s$, are good for the weight $w(x)=(1-x)^{\alpha} x^{\beta}(-\log x)^{\nu}$. In particular, the abscissas for the weight $w(x)=1$ are good also for the weights $w(x)=-\log x$, and $w(x)$ $=x^{\beta}(-\log x)$. Also, this property does not exist for Gaussian integration formulas. (As in (2), this property does not exist if instead of taking $R_{r}=1 /\left(r^{\alpha+\nu+1} z^{\eta}\right.$ ) we take $R_{r}=\mu_{r} / z^{r}$.)

(4) Our numerical quadrature formulas, in general, are about as efficient as the Gaussian formulas as the numerical results obtained over a set of different functions show; see Section 5.

We now give some properties of the new numerical quadrature formulas.

THEOREM 4.1. Let $I_{k}[f]$ in (1.7) be the numerical quadrature formula associated with $H_{k}(z)$ as given in (4.4). Then

$$
I_{k}\left[x^{i}\right]=I\left[x^{i}\right], \quad i=0,1, \ldots, k-1 .
$$

Proof. Using (3.1) in Theorem 2.2, it follows that

$$
H_{k}(z)=\sum_{j=1}^{k} \frac{\mu_{m}}{z^{m}}+O\left(\frac{1}{z^{k+1}}\right) \quad \text { as } z \rightarrow \infty ;
$$

and now the result follows as for the case of numerical quadrature formulas of interpolatory type which were described in Section 1.

Remark. For the weights $w(x)=(-\log x)^{\nu}, \nu>-1$, we have $\mu_{m}=\nu ! / m^{\nu+1}$, so that $R_{r}=\mu_{r} / z^{r}$. The corollary to Theorem 2.2 applies to this case, and we have

$$
H_{k}(z)=\sum_{j=1}^{k+1} \frac{\mu_{m}}{z^{m}}+O\left(\frac{1}{z^{k+2}}\right) \quad \text { as } z \rightarrow \infty
$$


From (4.8) it now follows that

$$
I_{k}\left[x^{i}\right]=I\left[x^{i}\right], \quad i=0,1, \ldots, k .
$$

The results in (4.8) and (4.9) hold also if we take $R_{r}=\mu_{r} / z^{r}$ instead of $R_{r}=$ $1 /\left(r^{\alpha+\nu+1} z^{\eta}\right)$ as in (4.2) and (4.3).

From (4.6) and (4.9) it follows that $I_{k}$ is a numerical quadrature formula of interpolatory type whose abscissas are not preassigned.

We now go on to investigate some properties of the poles of $T_{k, n}$ as defined in (4.2).

THEOREM 4.2. Let

$$
D_{k, n, m}(z)=\sum_{j=0}^{k}(-1)^{j}\left(\begin{array}{l}
k \\
j
\end{array}\right)(n+j)^{m} z^{n+j-1}, \quad m \geqslant 0 \text { integer. }
$$

Then $D_{k, n, m}(z)$ has $n+k-1$ zeros on $[0,1]$, such that $z=0$ is a zero of multiplicity $n-1, z=1$ is a zero of multiplicity $k-m$ only if $m \leqslant k-1$, and the rest are simple zeros in $(0,1)$. Furthermore, the simple zeros of $D_{k, n, m}(z)$ and those of $D_{k, n, m-1}(z)$, on $(0,1)$, interlace.

Proof. We start by observing that

$$
\begin{aligned}
D_{k, n, m}(z) & =\left(\frac{d}{d z} z\right)^{m} \sum_{j=0}^{k}(-1)^{j}\left(\begin{array}{l}
k \\
j
\end{array}\right) z^{n+j-1} \\
& =\left(\frac{d}{d z} z\right)^{m}\left[z^{n-1}(1-z)^{k}\right]
\end{aligned}
$$

Therefore,

$$
D_{k, n, m+1}(z)=\frac{d}{d z}\left[z D_{k, n, m}(z)\right], \quad m=0,1, \ldots .
$$

Now $D_{k, n, 0}(z)=z^{n-1}(1-z)^{k}$ has a zero of multiplicity $n-1$ at $z=0$ and another of multiplicity $k$ at $z=1$. Then by (4.12),

$$
D_{k, n, 1}(z)=\frac{d}{d z}\left[z D_{k, n, 0}(z)\right] \text {, }
$$

so that by Rolle's Theorem, $D_{k, n, 1}(z)$ has a zero of multiplicity $n-1$ at $z=0$ and one of multiplicity $k-1$ at $z=1$ and another simple zero $x_{1}^{1}$ in $(0,1) . D_{k, n, 2}(z)$, again by (4.12) and Rolle's Theorem, has a zero of multiplicity $n-1$ at $z=0$, one of multiplicity $k-2$ at $z=1$ and two simple zeros $x_{1}^{2}, x_{2}^{2}$ in $(0,1)$ one of them being in $\left(0, x_{1}^{1}\right)$, the other, in $\left(x_{1}^{1}, 1\right)$. Continuing this way we can show that $D_{k, n, m}(z)$ has a zero of multiplicity $n-1$ at $z=0$, one of multiplicity $k-m$ at $z=1$ and $m$ simple zeros on $(0,1)$, provided $m \leqslant k-1$. When $m=k-1, z=1$ is a simple zero, and therefore, for $m \geqslant k$ there is no zero at $z=1$. Consequently, for $m \geqslant k$, the number of simple zeros in $(0,1)$ is $k$. This way we also see that the simple zeros of $D_{k, n, m-1}(z)$ and $D_{k, n, m}(z)$ on $(0,1)$ interlace, starting with $x_{1}^{m}<x_{1}^{m-1}$. This completes the proof of the theorem.

As an immediate consequence of this theorem we have the following result:

COROllary. If $\alpha+\nu$ is a nonnegative integer, then $T_{k, 1}$ in (4.3) has $k$ simple poles in $(0,1)$. 
Now it is known that the zeros of two orthogonal polynomials of consecutive order have the interlacing property. It turns out that the polynomials $D_{k, n, m}(z)$ have a similar property. Before we give this result let us denote the number of the simple zeros of $D_{k, n, m}(z)$ on $(0,1)$ by $p$. According to the previous theorem if $m \leqslant k-1$, $p=m$ and if $m \geqslant k, p=k$ i.e., $p=\min (m, k)$. Let us denote these simple zeros by $0<x_{1}^{k, m}<x_{2}^{k, m}<\cdots<x_{p}^{k, m}<1$.

THEOREM 4.3. If $m \geqslant 2$, the simple zeros of $D_{k, n, m}(z)$ and $D_{k-1, n, m-1}(z)$ on $(0,1)$ have the interlacing property, i.e.,

$$
0<x_{1}^{k, m}<x_{1}^{k-1, m-1}<x_{2}^{k, m}<x_{2}^{k-1, m-1}<\cdots<x_{p-1}^{k-1, m-1}<x_{p}^{k, m}<1 .
$$

Proof. We start by writing (4.11) in the form

$$
\begin{aligned}
D_{k, n, m}(z) & =\left(\frac{d}{d z} z\right)^{m-1} \frac{d}{d z}\left[z^{n}(1-z)^{k}\right] \\
& =\left(\frac{d}{d z} z\right)^{m-1}\left[(n+k) z^{n-1}(1-z)^{k}-k z^{n-1}(1-z)^{k-1}\right] \\
& =(n+k) D_{k, n, m-1}(z)-k D_{k-1, n, m-1}(z) .
\end{aligned}
$$

If we let $z=x_{j}^{k, m}$ and $z=x_{j+1}^{k, m}(1 \leqslant j<p)$ in (4.14) and use the fact that $\nu_{k, n, m}(z)$ $=0$ for these values of $z$, we obtain

$$
k D_{k-1, n, m-1}\left(x_{i}^{k, m}\right)=(n+k) D_{k, n, m-1}\left(x_{i}^{k, m}\right), \quad i=j, j+1 .
$$

Now we showed in the previous theorem that the simple zeros of $D_{k, n, m}(z)$ and $D_{k, n, m-1}(z)$ on $(0,1)$ interlace. Therefore, $D_{k, n, m-1}\left(x_{i}^{k, m}\right) \neq 0, i=1, \ldots, p$; furthermore, $D_{k, n, m-1}\left(x_{j}^{k, m}\right)$ and $D_{k, n, m-1}\left(x_{j+1}^{k, m}\right)$ have opposite signs. This together with (4.15) implies that $D_{k-1, n, m-1}\left(x_{j}^{k, m}\right)$ and $D_{k-1, n, m-1}\left(x_{j+1}^{k, m}\right)$ have opposite signs, too. Therefore, $D_{k-1, n, m-1}(z)$ must vanish at least once in $\left(x_{j}^{k, m}, x_{j+1}^{k, m}\right)$. But since the number of simple zeros of $D_{k-1, n, m-1}(z)$ in $(0,1)$ is $p-1$, there can be at most one zero in $\left(x_{j}^{k, m}, x_{j+1}^{k, m}\right)$; and this completes the proof of the theorem.

COROllary. When $\alpha+\nu$ is a nonnegative integer, the poles of $T_{k, 1}$ and $T_{k-1,1}$ interlace in $(0,1)$.

Remark. Although we have proved that the $x_{k, j}$ are simple and lie in $(0,1)$ and that they have the interlacing property only for the case $\alpha+\nu$ a nonnegative integer, numerical results indicate that this holds for all $\alpha+\nu$ such that $\alpha+\nu>-1$. Numerical results also indicate that the weights $A_{k, j}$ are all positive, although no proof of this is available at the time of writing. Now the positiveness of the $A_{k, j}$ together with the result of Theorem 4.1 implies that $\lim _{k \rightarrow \infty} I_{k}[f]=I[f]$, for every function $f(x)$, continuous on $[0,1]$. This follows from Pólya's theorem on numerical quadrature; see Pólya (1933). The positiveness of the $A_{k, j}$ is also important numerically, for if the $A_{k, j}$ are of mixed sign, large losses of significance may take place by cancellation.

Finally, we shall give an exact expression for the error in case the integrand is analytic as described in Section 1. Combining (1.10) with (2.23), we have the follow- 
ing exact expression for the error

$$
E_{k}[f]=I[f]-I_{k}[f]=\frac{1}{2 \pi i} \int_{\Gamma} \frac{Q_{k, 1}(z)}{D_{k, 1}(z)} f(z) d z .
$$

Unfortunately, numerous efforts to find the asymptotic behavior of $Q_{k, 1}(z) / D_{k, 1}(z)$ as $k \rightarrow \infty$ have not been successful so far. However, as pointed out in Section 2 , this quotient, at least numerically speaking, goes to zero very quickly for all values of $z$ which makes $I_{k}[f]$ a very accurate numerical quadrature formula even for small $k$.

5. Numerical Examples. As mentioned in the previous section, the implementation of the new numerical quadrature rules for the weight functions in Table 3.1 is very simply achieved as we now explain. First we find the zeros $x_{k, i}$ of the denominator of $T_{k, 1}$, namely, we solve the polynomial equation, $\Sigma_{j=0}^{k} \lambda_{j} z^{j}=0$, where

$$
\lambda_{j}=(-1)^{j}\left(\begin{array}{l}
k \\
j
\end{array}\right)(j+1)^{k+\alpha+\nu-s}, \quad j=0,1, \ldots, k
$$

where $s$ is a small nonnegative integer like $0,1,2$. Once the $x_{k, i}$ are found we compute the weights $A_{k, i}$ using the formula in (4.5) which we give explicitly here:

$$
A_{k, i}=\frac{\sum_{j=1}^{k} \lambda_{j} x_{k, i}^{j}\left(\sum_{m=1}^{j} \mu_{m} / x_{k, i}^{m}\right)}{\sum_{j=1}^{k} \lambda_{j} j x_{k, i}^{j-1}}, \quad i=1, \ldots, k .
$$

All that one needs for computing these formulas is a good polynomial equation solver and a subprogram that computes the factorial (or gamma) function accurately for determining the $\mu_{m}$, both of which can be found in a reasonably good computer library. The gamma function, for instance, is a standard library function on the IBM-370 computer. Furthermore, the computer program that one has to write for solving the problem, judging from (5.1) and (5.2), is remarkably trivial, provided double or extended precision is used. If the $\mu_{m}$ are not known explicitly, then they can be computed numerically as follows: Making the change of variable $x=e^{-t}$ (see Section 3), $\mu_{m}$ can be expressed as

$$
\mu_{m}=\int_{0}^{\infty} t^{\alpha+\nu} e^{-(\beta+1) t} G(t) d t,
$$

where $G(t)=e^{-(m-1) t}\left(\left(1-e^{-t}\right) / t\right)^{\alpha}$ is a function that is regular at $t=0$ and behaves like $e^{-(m-1) t} t^{-\alpha}$ at infinity. Since $\beta+1>0$, making a further change of variable $(\beta+1) t=\tau$, we can approximate the infinite integral in (5.3) by Gauss-Laguerre quadrature associated with the generalized Laguerre polynomials $L_{n}^{(\alpha+\nu)}(\tau)$. Of course, other methods can also be employed in the approximation of the integral in (5.3).

We note however, that since the $\lambda_{j}$ in (5.1) alternate in sign and also become large as $k \rightarrow \infty$, for large $k$, a loss of significance may take place in the computation of the $x_{k, i}$ and hence the $A_{k, i}$. The same problem exists also for orthogonal polynomials in Gaussian integration, but this problem is overcome by using the 3-term recursion relation for the polynomials provided such a recursion relation is known explicitly. Even when the 3-term recursion relation is not known explicitly, it can be computed numerically as shown by Gautschi (1968), (1970) and others. For the polynomials $D_{k, 1, k}(z)$ no such recursion relation has been found so far. 


\section{TABLE 5.1}

The abscissas $x_{k, i}$ for the new numerical quadrature formulas $\int_{0}^{1} w(x) f(x) d x$ $\approx \sum_{i=1}^{k} A_{k, i} f\left(x_{k, i}\right)$, where $w(x)=(1-x)^{\alpha} x^{\beta}(-\log x)^{\nu}$ such that $\alpha+\nu$ is a small nonnegative integer like $0,1,2$. These $x_{k, i}$ are the roots of the polynomial equation $\Sigma_{j=0}^{k} \lambda_{j} z^{j}=0$ with $\lambda_{j}=(-1)^{j}\left(\begin{array}{l}k \\ j\end{array}\right)(j+1)^{k}, j=0,1, \ldots, k$. The weights $A_{k, i}$ can be computed from (5.2).

$$
\mathrm{k}=2
$$

$0.1504720765483788232775982 Q+00$

$0.7384168123405100656112906 Q+00$

$\mathrm{k}=3$

$0.4966471483058575305905226 Q-01$ $0.3733950309821642533051325 Q+00$ $0.8425652541872499936358153 Q+00$

$$
k=4
$$

$0.1799068499801418676799616 Q=01$ $0.1834165223808434598995189 Q+00$ $0.5414157204189524143060943 Q+00$ $0.8955770722021899390263906 Q+00$

$$
\mathrm{k}=5
$$

$0.6965462488720095999339260 Q-02$ $0.9279357371061748132102829 Q-01$ $0.3274158560864037341661747 \mathrm{Q}+00$ $0.6563187419838210706584532 Q+00$ $0.9258942258127421446039757 Q+00$

$$
\mathrm{k}=6
$$

0.2826962449669843567172046Q-02 $0.4862455152896217179348058 Q-01$ $0.1976882676470386134795882 Q+00$ $0.4503451521122742843529424 Q+00$ $0.7351670962539650939849607 Q+00$ $0.9447647096318862001844346 Q+0()$

$$
\mathrm{k}=7
$$

0.1187258545164058717677836Q-02 $0.2628663472239343247216861 Q-01$ $0.1210706424838506827348276 Q+00$ $0.3040908096207194258505372 Q+00$ $0.5483105637900638923877405 Q+00$ $0.7906485354805577158829337 Q+00$ $0.9572768818037839950791146 Q+00$

$$
\mathrm{k}=8
$$

$0.51151216701435651841231230 \div 03$ 0.1458573317963311020790610Q-01 $0.7546523540379770912044874 Q-01$ $0.2056995360684232874945901 Q+00$ $0.3998834536687977554256855 \mathrm{Q}+00$ $0.6250150234434242490768516 Q+00$ $0.8308072107358005732935312 Q+00$ $0.9659870403646759393069153 Q+00$ $\mathrm{k}=9$

$0.2247255954393555904923013 Q-03$

$0.8269188094627755378000373 Q-02$ $0.4785186222081606855590946 Q-01$

$0.1402987492166232486762045 Q+00$ $0.2900554492782025171212649 Q+00$ $0.4820003130318437765745138 Q+00$ $0.6851379193950804327653673 Q+00$ $0.8606574901008240488865763 Q+00$ $0.9722887040665427964516711 \mathrm{Q}+00$

$$
k=10
$$

$0.1002519058889115202504542 Q-03$ $0.4772351178865461685612723 \mathrm{Q}-02$ 0.3081710497452419278588480 Q-01 $0.9668389372266823582635567 Q-01$ $0.2108292927888059733206482 Q+00$ $0.3681879873486372138418079 Q+00$ $0.5510145152797366651435548 Q+00$ $0.7326572440917100235857143 Q+00$ $0.8833778455567603466710631 Q+00$ $0.9769924074477204492631444 Q+00$

$$
k=11
$$

$0.4527279518770788353534865 Q-04$ $0.2795480278297609540268378 Q-02$ $0.2012015530035912152540944 Q-01$ $0.6733379444645636049782197 Q-01$ $0.1540683692211295125287271 Q+00$ $0.2806977184599953977119152 Q+00$ $0.4379750731607002055234364 Q+00$ $0.6086538380237029376078756 Q+00$ $0.7706276021403855723338535 Q+00$ $0.9010352101154075441053256 Q+00$ $0.9805950222280231924986018 Q+00$

$$
\mathrm{k}=12
$$

$0.2064864876175711446099109 Q-04$ $0.1658119145275128110097481 Q-02$ $0.1329440070499037457225052 Q-01$ $0.4736615645452630247649566 Q-01$ $0.1133435389770539225618005 Q+00$ $0.2143947425636104262780328 Q+00$ $0.3465368850551303055200429 Q+\theta 0$ $0.4991909417699800878211610 Q+00$ $0.6568098047861667263044339 Q+00$ $0.8013203430670089168540922 Q+00$ $0.9150102643845972961189348 Q+00$ $0.9834146345679861200493482 Q+00$ 
In Table 5.1 we give the abscissas for the weight function $w(x)=1$, which are also good for $w(x)=x^{\beta}, \beta>-1$, by (2) of Section 4 and for $w(x)=x^{\beta}(-\log x)$ or more generally for $w(x)=(1-x)^{\alpha} x^{\beta}(-\log x)^{\nu}$, with $\alpha+\nu=0,1,2$, for example, by (3) of Section 4. These abscissas have been computed by taking $\lambda_{j}=(-1)^{j}\left(\begin{array}{c}k \\ j\end{array}\right)(j+1)^{k}$, $j=0,1, \ldots, k$, i.e., by taking $s=\alpha+\nu$, since $\alpha+\nu$ is a small nonnegative integer.

\section{TABLE 5.2}

$G_{k}$ and $S_{k}$ stand for the $k$-point Gaussian and new rules, respectively, with $w(x)=1$. The abscissas for $S_{k}$ are those given in Table 5.1. 0 means that $I_{k}[f]$ has at least 16 correct significant decimal digits. (If the computations are done using double precision on IBM-370 (word length approximately 16 decimal digits), round-off error sets in starting with $S_{8}$ for the function $f(x)=x /\left(e^{x}-1\right)$.)

\begin{tabular}{|c|c|c|c|c|c|}
\hline \multirow[b]{2}{*}{ Rule } & \multicolumn{5}{|c|}{$\left|I[f]-I_{k_{k}}[f]\right|$} \\
\hline & $\int_{0}^{1} \frac{d x}{1+x^{4}}$ & $\int_{0}^{1} \frac{d x}{1+x^{2}}$ & $\int_{0}^{1} \frac{d x}{1+x}$ & $\int_{0}^{1} \frac{d x}{1+e^{x}}$ & $\int_{0}^{1} \frac{x d x}{e^{x}-1}$ \\
\hline $\begin{array}{l}G_{2} \\
s_{2}\end{array}$ & $\begin{array}{l}7 \times 10^{-3} \\
4 \times 10^{-3}\end{array}$ & $\begin{array}{l}2 \times 10^{-3} \\
4 \times 10^{-3}\end{array}$ & $\begin{array}{l}1 \times 10^{-3} \\
1 \times 10^{-3}\end{array}$ & $\begin{array}{l}2 \times 10^{-5} \\
1 \times 10^{-4}\end{array}$ & $\begin{array}{l}7 \times 10^{-6} \\
3 \times 10^{-5}\end{array}$ \\
\hline $\begin{array}{l}\mathrm{G}_{4} \\
\mathrm{~S}_{4}\end{array}$ & $\begin{array}{l}2 \times 10^{-5} \\
4 \times 10^{-4} \\
\end{array}$ & $\begin{array}{l}5 \times 10^{-6} \\
5 \times 10^{-5}\end{array}$ & $\begin{array}{l}2 \times 10^{-6} \\
3 \times 10^{-7}\end{array}$ & $\begin{array}{l}1 \times 10^{-9} \\
3 \times 10^{-8}\end{array}$ & $\begin{array}{l}1 \times 10^{-11} \\
1 \times 10^{-8}\end{array}$ \\
\hline $\begin{array}{l}G_{6} \\
S_{6}\end{array}$ & $\begin{array}{l}5 \times 10^{-7} \\
2 \times 10^{-5}\end{array}$ & $\begin{array}{l}1 \times 10^{-8} \\
3 \times 10^{-7}\end{array}$ & $\begin{array}{l}7 \times 10^{-10} \\
2 \times 10^{-9}\end{array}$ & $\begin{array}{l}4 \times 10^{-14} \\
1 \times 10^{-10}\end{array}$ & $\begin{array}{c}0 \\
1 \times 10^{-12}\end{array}$ \\
\hline $\begin{array}{l}G_{8} \\
S_{8}\end{array}$ & $\begin{array}{l}7 \times 10^{-9} \\
5 \times 10^{-7}\end{array}$ & $\begin{array}{l}2 \times 10^{-11} \\
1 \times 10^{-9}\end{array}$ & $\begin{array}{l}6 \times 10^{-13} \\
4 \times 10^{-11}\end{array}$ & $\begin{array}{c}0 \\
1 \times 10^{-14}\end{array}$ & 0 \\
\hline $\begin{array}{l}G_{10} \\
s_{10} \\
\end{array}$ & $\begin{array}{l}4 \times 10^{-11} \\
1 \times 10^{-8}\end{array}$ & $\begin{array}{l}1 \times 10^{-13} \\
4 \times 10^{-11}\end{array}$ & $\begin{array}{l}6 \times 10^{-16} \\
1 \times 10^{-13}\end{array}$ & 0 & 0 \\
\hline $\begin{array}{l}G_{12} \\
s_{12} \\
\end{array}$ & $\begin{array}{l}2 \times 10^{-13} \\
2 \times 10^{-11} \\
\end{array}$ & $\begin{array}{l}1 \times 10^{-16} \\
5 \times 10^{-13}\end{array}$ & 0 & 0 & 0 \\
\hline Exact & $0.866972 \ldots$ & $0.785398 \ldots$ & $0.693147 \ldots$ & $0.379885 \ldots$ & $0.777504 .$. \\
\hline
\end{tabular}

In Tables 5.2, 5.3, 5.4, and 5.5 we compare the approximations $I_{k}[f]$, obtained by using the new rules and the Gaussian rules, with $w(x)=1, w(x)=x^{-1 / 2}, w(x)=$ $x^{-1 / 2}(-\log x)$, and $w(x)=(1-x)^{1 / 2} x^{-1 / 2}$, respectively. The abscissas of the new rules for the first three weight functions are the same and are those given in Table 5.1. The abscissas of the new rules for the fourth weight function are the zeros of the polynomials $\Sigma_{j=0}^{k} \lambda_{j} z^{j}$, where $\lambda_{j}=(-1)^{j}\left(\begin{array}{c}k \\ j\end{array}\right)(j+1)^{k+1 / 2}, j=0,1, \ldots, k$. Although Theorem 
4.2 does not apply to this case and to the cases for which $\lambda_{j}=(-1)^{j}\left({ }_{j}^{k}\right)(j+1)^{k+\alpha}$, in general, nevertheless, the abscissas are all real, distinct, and lie in $(0,1)$, and the weights are all positive, as numerous computations have shown. (We shall not give tables of these rules here.)

TABLE 5.3

$G_{k}$ and $S_{k}$ stand for the $k$-point Gaussian and new rules, respectively, with $w(x)=x^{-1 / 2}$. The abscissas for $S_{k}$ are those given in Table 5.1. 0 means that $I_{k}[f]$ has at least 16 correct significant decimal digits.

\begin{tabular}{|l|c|c|c|c|c|}
\hline \multirow{2}{*}{ Rule } & \multicolumn{5}{|c|}{$\left|I[f]-I_{k}[f]\right|$ for $][f]=\int_{0}^{1} x^{-\frac{1}{2}} f(x) d x$} \\
\cline { 2 - 6 } & $f(x)=\frac{1}{1+x^{4}}$ & $f(x)=\frac{1}{1+x^{2}}$ & $f(x)=\frac{1}{1+x}$ & $f(x)=\frac{1}{1+e^{x}}$ & $f(x)=\frac{x}{e^{x}-1}$ \\
\hline$G_{2}$ & $1 \times 10^{-2}$ & $2 \times 10^{-3}$ & $2 \times 10^{-3}$ & $4 \times 10^{-5}$ & $2 \times 10^{-5}$ \\
$S_{2}$ & $8 \times 10^{-3}$ & $2 \times 10^{-2}$ & $2 \times 10^{-2}$ & $6 \times 10^{-4}$ & $2 \times 10^{-3}$ \\
\hline$G_{4}$ & $1 \times 10^{-5}$ & $1 \times 10^{-5}$ & $2 \times 10^{-6}$ & $3 \times 10^{-9}$ & $3 \times 10^{-11}$ \\
$S_{4}$ & $5 \times 10^{-4}$ & $5 \times 10^{-5}$ & $3 \times 10^{-5}$ & $2 \times 10^{-7}$ & $1 \times 10^{-7}$ \\
\hline$G_{6}$ & $1 \times 10^{-6}$ & $2 \times 10^{-8}$ & $2 \times 10^{-9}$ & $1 \times 10^{-13}$ & 0 \\
$S_{6}$ & $2 \times 10^{-5}$ & $5 \times 10^{-7}$ & $2 \times 10^{-9}$ & $2 \times 10^{-12}$ & $3 \times 10^{-12}$ \\
\hline$G_{8}$ & $1 \times 10^{-8}$ & $5 \times 10^{-11}$ & $2 \times 10^{-12}$ & 0 & 0 \\
$S_{8}$ & $4 \times 10^{-7}$ & $3 \times 10^{-9}$ & $2 \times 10^{-11}$ & $3 \times 10^{-14}$ & 0 \\
\hline$G_{10}$ & $3 \times 10^{-11}$ & $1 \times 10^{-13}$ & $1 \times 10^{-15}$ & 0 & 0 \\
$S_{10}$ & $4 \times 10^{-9}$ & $2 \times 10^{-11}$ & $1 \times 10^{-13}$ & 0 & 0 \\
\hline$G_{12}$ & $7 \times 10^{-13}$ & 0 & 0 & 0 & 0 \\
\hline$s_{12}$ & $2 \times 10^{-10}$ & $5 \times 10^{-13}$ & 0 & 0 & 0 \\
\hline$E_{12 a c t}$ & $1.84930 .$. & $1.73394 \ldots$ & $1.57079 .$. & 0.838932. & $1.69969 \ldots$ \\
\hline
\end{tabular}

As can be seen from Tables 5.2-5.5, the new rules can, in general, compete favorably with the Gaussian rules, when applied to functions analytic in a domain containing the interval $[0,1]$. When the singularities of the integrands $f(x)$ are not too far away from the interval $[0,1]$ as, for example, for $f(x)=\left(1+x^{4}\right)^{-1}, f(x)=\left(1+x^{2}\right)^{-1}$ and $f(x)=(1+x)^{-1}$ in Tables 5.2-5.5, both rules converge quickly, the Gaussian rules being slightly better. For functions $f(x)$ whose singularities are far away from the interval $[0,1]$ as, for example, for $f(x)=1 /\left(1+e^{x}\right)$ and $f(x)=x /\left(e^{x}-1\right)$ in Tables 5.25.5 , the convergence of both rules becomes quicker, that of the Gaussian rules becoming even quicker.

The fact that the abscissas of the new rules that are given in Table 5.1 do not include the endpoints of the interval of integration (a property shared by the Gaussian in- 
tegration rules too), suggests that they can, like the Gaussian rules, be used in numerical integration by avoiding endpoint singularities, see Davis and Rabinowitz (1975, p. 144). It turns out that the new integration rules with $w(x)=1$ on $[0,1]$, work very efficiently on integrals of the form $\int_{0}^{1} f(x) d x$, where the functions $f(x)$ have algebraic or logarithmic singularities or products of them at $x=0$, especially when the $f(x)$ are

TABLE 5.4

$G_{k}$ and $S_{k}$ stand for the $k$-point Gaussian and new rules, respectively, with $w(x)=x^{-1 / 2}(-\log x)$. The abscissas for $S_{k}$ are those given in Table 5.1. 0 means that $S_{k}$ has at least 16 correct significant decimal digits. $G_{k}$ have been computed using the 12-figure tables of Boujot and Maroni (1968). 0* means that $G_{k}$ has at least 12 correct significant decimal digits.

\begin{tabular}{|c|c|c|c|c|c|}
\hline \multirow[b]{2}{*}{ Rule } & \multicolumn{5}{|c|}{$\left|I[f]-I_{k}[f]\right|$ for $I[f]=\int_{0}^{1} x^{-\frac{1}{2}}(-\log x) f(x) d x$} \\
\hline & $f(x)=\frac{1}{1+x^{4}}$ & $f(x)=\frac{1}{1+x^{2}}$ & $f(x)=\frac{1}{1+x}$ & $f(x)=\frac{1}{1+e^{x}}$ & $f(x)=\frac{x}{e^{x}-1}$ \\
\hline$G_{2}$ & $4 \times 10^{-4}$ & $5 \times 10^{-4}$ & $2 \times 10^{-3}$ & $2 \times 10^{-5}$ & $8 \times 10^{-6}$ \\
\hline$s_{2}$ & $1 \times 10^{-1}$ & $1 \times 10^{-1}$ & $1 \times 10^{-1}$ & $3 \times 10^{-3}$ & $2 \times 10^{-2}$ \\
\hline$G_{4}$ & $5 \times 10^{-5}$ & $8 \times 10^{-6}$ & $1 \times 10^{-6}$ & $1 \times 10^{-9}$ & $1 \times 10^{-11}$ \\
\hline$s_{4}$ & $4 \times 10^{-5}$ & $4 \times 10^{-5}$ & $3 \times 10^{-4}$ & $3 \times 10^{-6}$ & $1 \times 10^{-6}$ \\
\hline$G_{6}$ & $5 \times 10^{-7}$ & $1 \times 10^{-9}$ & $1 \times 10^{-9}$ & $0^{*}$ & $0^{\star}$ \\
\hline$s_{6}$ & $2 \times 10^{-6}$ & $2 \times 10^{-6}$ & $2 \times 10^{-7}$ & $1 \times 10^{-9}$ & $4 \times 10^{-11}$ \\
\hline$G_{8}$ & $2 \times 10^{-9}$ & $4 \times 10^{-11}$ & $0^{*}$ & $0^{*}$ & $0^{*}$ \\
\hline$s_{8}$ & $2 \times 10^{-7}$ & $6 \times 10^{-9}$ & $2 \times 10^{-10}$ & $1 \times 10^{-13}$ & 0 \\
\hline$G_{10}$ & $3 \times 10^{-11}$ & $0^{*}$ & $0^{*}$ & $0^{*}$ & $0^{\star}$ \\
\hline$s_{10}$ & $1 \times 10^{-8}$ & $8 \times 10^{-11}$ & $3 \times 10^{-13}$ & 0 & 0 \\
\hline$G_{12}$ & $0^{*}$ & $0^{*}$ & $0^{\star}$ & $0^{\star}$ & $0^{*}$ \\
\hline$s_{12}$ & $5 \times 10^{-10}$ & $4 \times 10^{-14}$ & $1 \times 10^{-15}$ & 0 & 0 \\
\hline Exact & $3.96017 \ldots$ & $3.874184 \ldots$ & 3.663862 . & $1.890524 \ldots$ & 3.791043 \\
\hline
\end{tabular}

continuous at $x=0$. For such integrals, numerous computations have shown that the new rules are superior to the Gaussian rules. A comparison of Gaussian and new rules with $w(x)=1$ on $[0,1]$ for the functions $f(x)=x^{-1 / 2}, f(x)=\log x, f(x)=x^{1 / 2} \log x$, $f(x)=x^{1 / 2}$, and $f(x)=x^{3 / 2}$, is given in Table 5.6. Note that the first function is the most singular, the second function is less singular, the third even less, etc. 
For $\lambda_{j}=(-1)^{j}\left({ }_{j}^{k}\right)(j+1)^{k}$ all the abscissas $x_{k, i}$ are in $(0,1)$ as mentioned before. By (3) of Section 4, we can take

$$
\lambda_{j}=(-1)^{j}\left(\begin{array}{l}
k \\
j
\end{array}\right)(j+1)^{k-1}, \quad j=0,1, \ldots, k,
$$

affecting very little the accuracy of $T_{k, n}$ hence that of $I_{k}[f]$. This time, however, the endpoint $z=1$ is an abscissa by Theorem 4.2. Thus, we have obtained accurate numerical integration rules similar to the Radau rules, see Davis and Rabinowitz (1975, pp. 79-80). The performance of these rules is demonstrated through two examples in Table 5.7, where $f(x)=(1+x)^{-1}$. (We shall not give tables of these rules here.)

\section{TABLE 5.5}

$G_{k}$ and $S_{k}$ stand for the $k$-point Gaussian and new rules, respectively, with $w(x)=(1-x)^{1 / 2} x^{-1 / 2}$. The abscissas of $S_{k}$ are the zeros of the polynomial $\sum_{j=0}^{k} \lambda_{j} z^{j}$, where $\lambda_{j}=(-1)^{j}\left({ }_{j}^{k}\right)(j+1)^{k+1 / 2} .0$ means that $I_{k}[f]$ has at least 16 correct significant decimal digits.

\begin{tabular}{|c|c|c|c|c|c|}
\hline \multirow[b]{2}{*}{ Rule } & \multicolumn{5}{|c|}{$\left|I[f]-I_{k}[f]\right|$ for $I[f]=\int_{a}^{1}(1-x)^{\frac{1}{2}} x^{-\frac{1}{2}} f(x) d x$} \\
\hline & $f(x)=\frac{1}{1+x^{4}}$ & $f(x)=\frac{1}{1+x^{2}}$ & $f(x)=\frac{1}{1+x}$ & $f(x)=\frac{1}{1+e^{x}}$ & $f(x)=\frac{x}{e^{x}-1}$ \\
\hline$G_{2}$ & $4 \times 10^{-3}$ & $6 \times 10^{-4}$ & $1 \times 10^{-3}$ & $2 \times 10^{-5}$ & $8 \times 10^{-6}$ \\
\hline$s_{2}$ & $5 \times 10^{-3}$ & $6 \times 10^{-2}$ & $6 \times 10^{-3}$ & $3 \times 10^{-4}$ & $1 \times 10^{-3}$ \\
\hline$G_{4}$ & $3 \times 10^{-5}$ & $8 \times 10^{-6}$ & $1 \times 10^{-6}$ & $1 \times 10^{-9}$ & $2 \times 10^{-11}$ \\
\hline$s_{4}$ & $2 \times 10^{-4}$ & $4 \times 10^{-5}$ & $1 \times 10^{-5}$ & $4 \times 10^{-8}$ & $3 \times 10^{-8}$ \\
\hline$G_{6}$ & $6 \times 10^{-7}$ & $8 \times 10^{-9}$ & $1 \times 10^{-9}$ & $5 \times 10^{-14}$ & 0 \\
\hline$s_{6}$ & $2 \times 10^{-5}$ & $2 \times 10^{-7}$ & $5 \times 10^{-10}$ & $4 \times 10^{-11}$ & $2 \times 10^{-13}$ \\
\hline$G_{8}$ & $5 \times 10^{-9}$ & $3 \times 10^{-11}$ & $1 \times 10^{-12}$ & 0 & 0 \\
\hline$s_{8}$ & $3 \times 10^{-6}$ & $1 \times 10^{-9}$ & $2 \times 10^{-11}$ & $2 \times 10^{-15}$ & 0 \\
\hline$G_{10}$ & $3 \times 10^{-12}$ & $6 \times 10^{-14}$ & 0 & 0 & 0 \\
\hline$s_{10}$ & $1 \times 10^{-8}$ & $3 \times 10^{-11}$ & $1 \times 10^{-13}$ & 0 & 0 \\
\hline$G_{12}$ & $5 \times 10^{-13}$ & 0 & 0 & 0 & 0 \\
\hline$s_{12}$ & $1 \times 10^{-10}$ & $4 \times 10^{-13}$ & 0 & 0 & 0 \\
\hline Exact & $1.507274 \ldots$ & 1.429706 & 1.301 & 0.689653 & 1.390 \\
\hline
\end{tabular}

6. Numerical Quadrature Using $T_{k, 2}$ : The Case $n=2$. Here we briefly discuss the case $n=2$ which was mentioned in Section 4. As can be seen from (4.2) and the discussion following (4.2), the denominator of $T_{k, 2}$ is a polynomial of degree $k+1$ with a simple zero at $z=0$, and the numerator is a polynomial of degree $\leqslant k$, such 
that the numerical quadrature formula obtained from $T_{k, 2}$ is a $(k+1)$-point rule. Having one of the endpoints $(z=0)$ as an abscissa, these are rules of Radau type.

Letting $H_{k}(z)=T_{k-1,2}$ and making use of Theorem 2.2 , we can see easily that the results of Theorem 4.1 and the remark following Theorem 4.1 apply to the numerical quadrature rules obtained from this new $H_{k}(z)$. Similarly the corollaries to Theorems 4.2 and 4.3 apply too. That is, when $\alpha+\nu$ is a nonnegative integer, $T_{k-1,2}$ has $k$ simple poles in $[0,1]$, one of them being at $z=0$ for all $k$; and the poles of $T_{k-2,2}$ and $T_{k-1,2}$ interlace on $(0,1)$.

\section{TABLE 5.6}

$G_{k}$ and $S_{k}$ stand for the $k$-point Gaussian and new rules, respectively, with $w(x)=1$; i.e., the singularity of $f(x)$ at $x=0$ is ignored. The abscissas for $S_{k}$ are those given in Table 5.1.

\begin{tabular}{|c|c|c|c|c|c|}
\hline \multirow[b]{2}{*}{ Rule } & \multicolumn{5}{|c|}{$\left|I[f]-I_{k}[f]\right|$} \\
\hline & $\int_{0}^{1} x^{-\frac{1}{2}} d x$ & $\int_{0}^{1} \log x d x$ & $\int_{0}^{1} x^{\frac{1}{2}} \log x d x$ & $\int_{0}^{1} x^{\frac{1}{2}} d x$ & $\int_{0}^{1} x^{3 / 2} d x$ \\
\hline$G_{2}$ & $3 \times 10^{-1}$ & $1 \times 10^{-1}$ & $2 \times 10^{-2}$ & $7 \times 10^{-3}$ & $1 \times 10^{-3}$ \\
\hline $\mathrm{S}_{2}$ & $3 \times 10^{-1}$ & $5 \times 10^{-2}$ & $1 \times 10^{-2}$ & $3 \times 10^{-3}$ & $9 \times 10^{-4}$ \\
\hline$G_{4}$ & $2 \times 10^{-1}$ & $3 \times 10^{-2}$ & $4 \times 10^{-3}$ & $1 \times 10^{-3}$ & $5 \times 10^{-5}$ \\
\hline$S_{4}$ & $6 \times 10^{-2}$ & $6 \times 10^{-4}$ & $5 \times 10^{-4}$ & $2 \times 10^{-4}$ & $7 \times 10^{-6}$ \\
\hline$a_{6}$ & $1 \times 10^{-1}$ & $1 \times 10^{-2}$ & $2 \times 10^{-3}$ & $4 \times 10^{-4}$ & $7 \times 10^{-6}$ \\
\hline$s_{6}$ & $1 \times 10^{-2}$ & $9 \times 10^{-4}$ & $8 \times 10^{-j}$ & $8 \times 10^{-6}$ & $5 \times 10^{-7}$ \\
\hline$C_{8}$ & $1 \times 10^{-1}$ & $2 \times 10^{-3}$ & $1 \times 10^{-3}$ & $2 \times 10^{-4}$ & $2 \times 10^{-6}$ \\
\hline$s_{8}$ & $5 \times 10^{-4}$ & $2 \times 10^{-4}$ & $1 \times 10^{-5}$ & $3 \times 10^{-6}$ & $4 \times 10^{-9}$ \\
\hline$s_{10}$ & $8 \times 10^{-2}$ & $6 \times 10^{-3}$ & $5 \times 10^{-4}$ & $9 \times 10^{-5}$ & $7 \times 10^{-7}$ \\
\hline$s_{10}$ & $2 \times 10^{-3}$ & $2 \times 10^{-5}$ & $5 \times 10^{-6}$ & $7 \times 10^{-7}$ & $5 \times 10^{-9}$ \\
\hline$G_{12}$ & $7 \times 10^{-2}$ & $4 \times 10^{-3}$ & $3 \times 10^{-4}$ & $6 \times 10^{-5}$ & $3 \times 10^{-7}$ \\
\hline$s_{12}$ & $1 \times 10^{-3}$ & $2 \times 10^{-5}$ & $4 \times 10^{-7}$ & $4 \times 10^{-3}$ & $2 \times 10^{-11}$ \\
\hline Exact & 2 & -1 & $4 / 9$ & $2 / 3$ & $2 / 5$ \\
\hline
\end{tabular}

Several computations have been done using the numerical quadrature formulas obtained from $T_{k, 2}$. The results of these computations indicate that the accuracy of these rules is practically the same as that of the numerical quadrature rules obtained from $T_{k, 1}$. 


\section{TABLE 5.7}

$S_{k}$ and $\overline{S_{k}}$ are the new $k$-point rules whose abscissas are the roots of the polynomial equation $\Sigma_{j=0}^{k} \lambda_{j} z^{j}=0$, where $\lambda_{j}=(-1)^{j}\left(\begin{array}{l}k \\ j\end{array}\right)(j+1)^{k}$ for $S_{k}$ and $\lambda_{j}=(-1)\left(\begin{array}{l}k \\ j\end{array}\right)(j+1)^{k-1}$ for $\overline{S_{k}}$. For the first column $w(x)=1$ and for the second column $w(x)=x^{-1 / 2} . \quad z=1$ is an abscissa for the rules $\overline{S_{k}}$.

\begin{tabular}{|l|l|c|}
\hline \multirow{2}{*}{ Rule } & $\left|I[f]-I_{k}[f]\right|$ & for the integrals \\
\cline { 2 - 3 } & $\int_{0}^{1} \frac{d x}{1+x}$ & $\int_{0}^{1} \frac{1}{\sqrt{x}} \frac{d x}{1+x}$ \\
\hline$S_{2}$ & $1 \times 10^{-3}$ & $2 \times 10^{-2}$ \\
$\bar{S}_{2}$ & $6 \times 10^{-3}$ & $7 \times 10^{-2}$ \\
\hline$S_{4}$ & $3 \times 10^{-3}$ & $3 \times 10^{-5}$ \\
$\bar{S}_{4}$ & $5 \times 10^{-6}$ & $8 \times 10^{-5}$ \\
\hline$S_{6}$ & $2 \times 10^{-9}$ & $3 \times 10^{-9}$ \\
$\bar{S}_{6}$ & $5 \times 10^{-8}$ & $2 \times 10^{-7}$ \\
\hline$S_{8}$ & $4 \times 10^{-11}$ & $2 \times 10^{-11}$ \\
$\bar{S}_{8}$ & $1 \times 10^{-10}$ & $2 \times 10^{-10}$ \\
\hline$S_{10}$ & $1 \times 10^{-13}$ & $1 \times 10^{-13}$ \\
$\bar{S}_{10}$ & $3 \times 10^{-13}$ & $1 \times 10^{-12}$ \\
\hline$E_{6 x a c t}$ & $0.693147 \ldots 7963 \ldots$ \\
\hline
\end{tabular}

For the sake of completeness we give here the coefficients of the polynomial $\Sigma_{j=0}^{k} \lambda_{j} z^{j+1}$ whose zeros are the abscissas $x_{k+1, i}, i=1, \ldots, k+1$ :

$$
\lambda_{j}=(-1)^{j}\left(\begin{array}{l}
k \\
j
\end{array}\right)(j+2)^{k+\alpha+\nu-s}, \quad j=0,1, \ldots, k
$$

where $s$ is a small nonnegative integer like $0,1,2$. We take $x_{k+1,1}=0$. The corresponding weights are then

$$
A_{k+1, i}=\frac{\sum_{j=0}^{k} \lambda_{j} x_{k+1, i}^{j}\left(\sum_{m=1}^{j+1} \mu_{m} / x_{k+1, i}^{m}\right)}{\sum_{j=0}^{k} \lambda_{j}(j+1) x_{k+1, i}^{j}}, \quad i=2, \ldots, k+1,
$$

with

$$
A_{k+1,1}=\sum_{j=0}^{k} \lambda_{j} \mu_{j} / \lambda_{0}
$$

Finally, if the $\lambda_{j}$ are taken to be

$$
\lambda_{j}=(-1)^{j}\left(\begin{array}{l}
k \\
j
\end{array}\right)(j+2)^{k-1}, \quad j=0,1, \ldots, k
$$


(for $w(x)=x^{\beta}, \beta>-1$, for example), then $z=1$ is also an abscissa; and hence, the new rule has both of its endpoints as abscissas, thus becoming a Lobatto-type rule, see Davis and Rabinowitz (1975, pp. 79-80).

7. Symmetric Rules. As can be seen from Theorem 4.2 and Table 5.1, the abscissas $x_{k, j}$ are not symmetric with respect to $x=1 / 2$ for $w(x)=1$ or for any weight function which is symmetric with respect to $x=1 / 2$. Actually, Table 5.1 indicates that as $k$ becomes large many of the $x_{k, j}$ cluster about $x=0$. We now show how symmetric rules can be obtained. Consider the integral

$$
I[f]=\int_{-1}^{+1} w(x) f(x) d x,
$$

where $w(x)$ is an even weight function, and define

$$
\mu_{m}=\int_{-1}^{+1} w(x) x^{2(m-1)} d x, \quad m=1,2, \ldots
$$

Then

$$
H(z)=\int_{-1}^{+1} \frac{w(x)}{z-x} d x=\sum_{m=1}^{\infty} \frac{\mu_{m}}{z^{2 m-1}}
$$

If we now let

$$
A_{0}=0, \quad A_{r}=\sum_{m=1}^{r} \frac{\mu_{m}}{z^{2 m-1}}, \quad r=1,2, \ldots
$$

and

$$
R_{r}=c_{r} / z^{2 r-1}, \quad r=1,2, \ldots,
$$

in (2.2), and simplify the rational function that is obtained, we can see that $T_{k, n}$ can have simple poles only if $n=1$ or $n=2$. When $n=1$, the numerator of $T_{k, 1}$ is an odd polynomial of degree $2 k-1$, and its denominator is an even polynomial of degree $2 k$. Therefore, $T_{k, 1}$ can provide us with a $2 k$-point numerical quadrature formula. When $n=2$, on the other hand, the numerator of $T_{k, 2}$ is an even polynomial of degree $2 k$, and its denominator is an odd polynomial of degree $2 k+1$. Therefore, $T_{k, 2}$ can provide us with a $(2 k+1)$-point numerical quadrature formula. It is seen easily that for these rules if $\xi$ is an abscissa, so is $-\xi$, and their corresponding weights are the same.

As an example, we shall consider the case $w(x)=1$. For this case $\mu_{m}=$ $2 /(2 m-1), m=1,2, \ldots$. Therefore, we choose $R_{r}=1 /\left(r z^{2 r-1}\right), r=1,2, \ldots$. The denominator of $T_{k, 1}$ becomes $\Sigma_{j=0}^{k} \lambda_{j} z^{2 j}$, where

$$
\lambda_{j}=(-1)^{j}\left(\begin{array}{l}
k \\
j
\end{array}\right)(j+1)^{k}, \quad j=0,1, \ldots, k ;
$$

and the denominator of $T_{k, 2}$ becomes $\Sigma_{j=0}^{k} \lambda_{j} z^{2 j+1}$, where

$$
\lambda_{j}=(-1)^{j}\left(\begin{array}{l}
k \\
j
\end{array}\right)(j+2)^{k}, \quad j=0,1, \ldots, k
$$

Using Theorem 4.2, we can see that all the poles of $T_{k, 1}$ and $T_{k, 2}$ are in $(-1,1)$. As before, by replacing $(j+1)^{k}$ in $(7.6)$ by $(j+1)^{k-1}$ and $(j+2)^{k}$ in (7.7) by 
$(j+2)^{k-1}$, we can make the endpoints $z= \pm 1$ also abscissas, thus obtaining Lobattotype formulas.

Now making use of Theorem 2.2 we can conclude that the numerical quadrature formulas obtained from $T_{k, 1}$ and $T_{k, 2}$ using (7.6) and (7.7) satisfy

$$
I_{2 k}\left[x^{i}\right]=I\left[x^{i}\right], \quad i=0,1, \ldots, 2 k-1, k=1,2, \ldots,
$$

and

$$
I_{2 k+1}\left[x^{i}\right]=I\left[x^{i}\right], \quad i=0,1, \ldots, 2 k+1, k=1,2, \ldots
$$

If, however, we let $R_{r}=2 /\left[(2 r-1) z^{2 r-1}\right]$, then making use of the corollary to Theorem 2.2 we can replace (7.8) and (7.9) by

$$
\begin{gathered}
I_{2 k}\left[x^{i}\right]=I\left[x^{i}\right], \quad i=0,1, \ldots, 2 k+1, k=1,2, \ldots, \\
I_{2 k+1}\left[x^{i}\right]=I\left[x^{i}\right], \quad i=0,1, \ldots, 2 k+3, k=1,2, \ldots .
\end{gathered}
$$

Several computations using these methods have also been done. Their performance is very similar to the performance of the rules that use the same number of abscissas, given in the previous sections.

Let us finally consider the symmetric weight function $w(x)=\left(1-x^{2}\right)^{\alpha}$ on $[-1,1]$ which contains algebraic singularities at both endpoints. An analysis similar to that given in Section 3 shows that $R_{r}=1 /\left(r^{1+\alpha} z^{2 r-1}\right)$, i.e., there is no way of getting rid of the $\alpha$-dependence in the $R_{r}$. This implies that for different $\alpha$ 's different sets of abscissas are needed unlike some of the rules of the previous section.

8. Concluding Remarks. We have reviewed some nonlinear transformations for accelerating the convergence of infinite sequences due to Levin and used a modification of them to obtain numerical quadrature formulas for weight functions with algebraic and/or logarithmic endpoint singularities. These rules are simpler to compute than the corresponding Gaussian rules and practically as efficient as them. They also have the advantage that a whole family of weight functions can have the same set of abscissas, which we believe should be of practical importance. We have also shown how different numerical quadrature rules (of the Lobatto and Radau type) can be obtained. We have proved some properties for some of these new rules although our theory is not complete. However, all the numerical computations that we have done indicate that these rules are good integration rules, i.e., all their abscissas are distinct and lie in the interval of integration, and all their weights are positive. It is hoped to contribute further to the theory of these new numerical integration rules in the future.

Acknowledgement. The author wishes to thank Dr. D. Levin and Professor P. Rabinowitz for useful discussions and comments. The computations for this paper were carried out on the IBM-370 computer at the Computation Center of the Technion, Israel Institute of Technology. 
G. BAKER, JR. (1975), Essentials of Padé Approximants, Academic Press, New York.

J. P. BOUJOT \& P. MARONI (1968), Algorithme Général de Construction de Tables de Gauss pour les Problèmes de Quadratures, Institut Blaise Pascal, publication No. MMX/8.1.8/AI.

P. J. DAVIS (1955), "On a problem in the theory of mechanical quadratures," Pacific J. Math., v. 5, pp. 669-674.

P. J. DAVIS \& P. RABINOWITZ (1975), Methods of Numerical Integration, Academic Press, New York.

W. GAUTSCHI (1968), "Construction of Gauss-Christoffel quadrature formulas," Math. Comp., v. 22, pp. 251-270.

W. GAUTSCHI (1970), "On the construction of Gaussian quadrature rules from modified moments," Math. Comp., v. 24, pp. 245-260.

G. H. GOLUB \& J. H. WELSCH (1969), "Calculation of Gauss quadrature rules," Math. Comp., v. 23, pp. $221-230$.

D. LEVIN (1973), "Development of non-linear transformations for improving convergence of sequences," Internat. J. Comput. Math., v. B3, pp. 371-388.

D. LEVIN \& A. SIDI (1975), "Two new classes of non-linear transformations for accelerating the convergence of infinite integrals and series," Appl. Math. Comput. (To appear.)

I. M. LONGMAN (1973), "On the generation of rational approximations for Laplace transform inversion with an application to viscoelasticity," SIAM J. Appl. Math., v. 24, pp. 429-440.

F. W. J. OLVER (1974), Asymptotics and Special Functions, Academic Press, New York. 264-286.

G. PÓLYA (1933), “Über die Konvergenz von Quadraturverfahren," Math. Z., v. 37, pp.

D. SHANKS (1955), "Non-linear transformations of divergent and slowly convergent sequences," J. Math. and Phys., v. 34, pp. 1-42.

A. SIDI (1979), "Convergence properties of some nonlinear sequence transformations," Math. Comp., v. 33, pp. 315-326. 